\title{
Anesthesia-Related Risk Factors for Anterolateral Thigh Free Flap Complications in 161 Elderly Chinese Patients Who Received Oral and Maxillofacial Reconstruction
}

\author{
Chengli Wang, $M D^{1,2}$, Zhixiao Han, $M D^{1,2}$, Shi Cheng, $M D^{1,2}$ and Minghui Cao, $M D, P h D^{1,2^{*}}$ \\ ${ }^{1}$ Guangdong Provincial Key Laboratory of Malignant Tumor Epigenetics and Gene Regulation, Sun Yat-Sen Memorial \\ Hospital, Sun Yat-Sen University, Guangzhou, China \\ ${ }^{2}$ Department of Anesthesiology, Sun Yat-sen Memorial Hospital, Sun Yat-sen University, Guangzhou, China
}

\begin{abstract}
Purpose: To determine anesthesia-related risk factors that predicted complications in elderly Chinese patients undergoing oral and maxillofacial reconstruction with Anterolateral Thigh Free Flap (ATFF).

Patients and methods: The authors designed and implemented a retrospective study which enrolled patients at over 65-years-old who underwent resection of oral and maxillofacial tumors and ATFF reconstruction from January 2011 to March 2019. Predictor variables were divided into: 1) Demographic variables (gender; age; weight; comorbidities; smoking status); 2) Anesthetic (American Society of Anesthesiologists (ASA) classification; preoperative and postoperative hemoglobin and albumin level, blood loss, blood transfusion, urine output ( $\mathrm{ml}$ ), and infusion of crystalloids and colloids, and volumes given intraoperatively and postoperatively for 24 hours). The primary outcome was the presence of postoperative complications (yes/no), and the secondary outcome was types of complications (medical and surgical). All the variables were analyzed by univariate and multivariable analysis and statistical significance was set at a $\mathrm{P}<0.05$.
\end{abstract}

Results: The study sample was composed of 161 patients with a mean age of 69 years. 31 patients had postoperative complications, and 3 of whom had total flap necrosis. Risk factors were: Postoperative hypoproteinemia and ASA grade.

Conclusions: For patients over 65-years-old accepting reconstruction with a ATFF, postoperative hypoproteinemia and ASA grade are potential anesthesia-related predictors of postoperative complications.

The occurrence of oral and maxillofacial tumors in elderly patients is gradually increasing worldwide with a quickly developing aging society [1]. In the United States, among all oral and maxillofacial malignancies, $25 \%$ of which are reckoned to happen in the elderly [2]. The most common treatment for these patients is surgical resection. However, this kind of treatment may have a large impact on the structure and lead to dysfunction of the oral and maxillofacial regions. As we know, elderly patients usually have a variety of systemic diseases. It is especially important for them to have the defects reconstructed with a flap, which can promote complete or partial recovery of both function and aesthetics, so that they can regain their health. Various donor sites are available for reconstructing the defects, including medial calf flap, anterolateral thigh free flap, and anterolateral thigh free flap. However, regardless of which flap was chosen, postoperative complications were still an important factor influencing prognosis.

In the present study, we hypothesized that ATFF complications could be susceptible to some perioperative variables. The aim of this study was to determine the potential perioperative risk factors especially those associated with anesthesia that lead to postoperative complications in elderly Chinese patients who received reconstruction with ATFF. The specific aims of the study were: 1) To gather clinical data about elderly Chinese patients who received reconstructions of the oral and maxillofacial defects by ATFF over the past 9 years; 2 ) To analyze the associations among perioperative variables, especially those which are

*Corresponding author: Dr. Minghui Cao, MD, PhD, Department Head of Department of Anesthesiology, Sun Yat-sen Memorial Hospital, Sun Yat-sen University, No. 107, Yanjiang West Road, Guangzhou, 510120, China, Tel: +86-1892-218-2538, Fax: 0086-20-8133-2283

Accepted: September 28, 2019

Published online: September 30, 2019

Citation: Wang C, Han Z, Cheng S, et al. (2019) Anesthesia-Related Risk Factors for Anterolateral Thigh Free Flap Complications in 161 Elderly Chinese Patients Who Received Oral and Maxillofacial Reconstruction. J Head Neck Surg 2(1):33-37 
anesthesia related ATFF; 3) To estimate the effect of anesthesia-related risk factors on the development of early postoperative complications of the ATFF.

\section{Patients and Methods}

\section{Retrospective study and sample}

To solve the research purpose, the investigators designed and implemented a retrospective study. The study population was composed of all elderly patients presenting to the Oral and Maxillofacial Surgery Department of Sun Yat-sen Memorial Hospital, Sun Yat-sen University (Guangzhou, China) for evaluation and management of resection of oral and maxillofacial tumors and anterolateral thigh free flap reconstruction from January 2011 to March 2019. To be included in the study sample, patients had to be at least 65 -years-old. Study approval was obtained from the Institutional Review Board (IRB) of Sun Yat-sen Memorial Hospital of Sun Yat-sen University. As the work belonged to retrospectively review medical records and was certified by the IRB as low risk, informed consent was not required by the IRB.

\section{Variables}

The main predictors were grouped into two groups according to our previous study [3]: 1) Demographic variables, including gender, age, weight, smoking status and coexisting diseases (hypertension, diabetes mellitus and heart disease). 2) Anesthetic variables (American Society of Anesthesiologists (ASA) classification, preoperative and postoperative hemoglobin and albumin level, blood loss, blood transfusion, urine output ( $\mathrm{ml}$ ) and infusion of crystalloids and colloids, and volumes given intraoperatively and postoperatively for 24 hours). Flap complications (yes/ no) during the postoperative period, were designated the primary outcome variable. The secondary outcome variable was types of complications (medical and surgical). The surgical postoperative complications were any complication that occurred at the donor or recipient site, which included necrosis or partial necrosis, swelling, ecchymosis, dehiscence, exudation, and exploration. The assessed postoperative medical complications included electrolyte disturbances, hyperglycemia, dysphoria, drug-induced liver injury, myocardial infarction, renal insufficiency, and pneumonia.

\section{Data analyses}

Univariate analysis and multivariate analysis were performed using the logistic regression model to assess the significance of the risk factors for complications. A $P$ value less than 0.05 was considered statistically significant. All statistical analyses were performed using IBM SPSS Statistics for Windows software (version 22, IBM Corp, Armonk, NY, USA).

\section{Results}

\section{Demographic and anesthetic variables}

Patient demographic and clinical characteristics are listed in Table 1. There were 65 female and 96 male cases, with a mean age of $69.1 \pm 3.6$ years (range, 65 to 80 years).
A review of the anesthesia records showed that $45.3 \%$ of all patients belonged to American Society of Anesthesiologists categories 3 , and $32.3 \%$ of patients had 1 or more prognostic comorbidities. The mean preoperative Hgb level was $13.5 \mathrm{~g} / \mathrm{dL}$ (range, 8.1 to $17.5 \mathrm{~g} / \mathrm{dL}$ ), and the mean preoperative albumin level was $40.6 \mathrm{~g} / \mathrm{L}$ (range, 30.1 to $49.4 \mathrm{~g} / \mathrm{L}$ ). The mean postoperative $\mathrm{Hgb}$ level was $10.9 \mathrm{~g} /$ $\mathrm{dL}$ (range, 7.0 to $14.8 \mathrm{~g} / \mathrm{dL}$ ), and the mean postoperative albumin level was $32.2 \mathrm{~g} / \mathrm{L}$ (range, 23.1 to $38.5 \mathrm{~g} / \mathrm{L}$ ). Blood transfusions were administered in 28 patients (17.4\%).

\section{Incidence of complications}

In total, we identified 161 patients who underwent Table 1: Demographic and clinical characteristics.

\begin{tabular}{|l|l|}
\hline Variables & All patients $(\mathbf{n}=\mathbf{1 6 1})$ \\
\hline Mean (range) age (years) & $69(65-80)$ \\
\hline Mean (range) weight (kg) & $60(37-97)$ \\
\hline Sex: & 96 \\
\hline Male & 65 \\
\hline Female & \\
\hline ASA score: & 88 \\
\hline II & 73 \\
\hline III & \\
\hline Smoking: & 64 \\
\hline Yes & 97 \\
\hline No & 109 \\
\hline No comorbidities & \\
\hline Comorbidities & 21 \\
\hline Hypertension & 11 \\
\hline Diabetes & 20 \\
\hline Both & \\
\hline
\end{tabular}

Abbreviations: ASA: American Society of Anesthesiologists.

Table 2: Types of flap complications.

\begin{tabular}{|l|l|}
\hline Complications & n \\
\hline Surgical: & \\
\hline Partial flap necrosis & 8 \\
\hline Total flap necrosis & 3 \\
\hline Haematoma & 2 \\
\hline Flap infection & 2 \\
\hline Ecchymosis & 2 \\
\hline Dehiscence & 1 \\
\hline Total & 18 \\
\hline Medical: & \\
\hline Electrolyte disturbance & 4 \\
\hline Pneumonia & 15 \\
\hline Renal dysfunction & 1 \\
\hline Total & 20 \\
\hline Both medical and surgical & 7 \\
\hline
\end{tabular}


Citation: Wang C, Han Z, Cheng S, et al. (2019) Anesthesia-Related Risk Factors for Anterolateral Thigh Free Flap Complications in 161 Elderly Chinese Patients Who Received Oral and Maxillofacial Reconstruction. J Head Neck Surg 2(1):33-37

161 microsurgical anterolateral thigh free flap procedures. Outcome measures obtained from the complete dataset of 161 cases were 30 -day mortality rate and complications. In the present study, no patients died during the 30-day postoperative period. Postoperative in-hospital complications developed in 31 of 161 patients (19.3\%). There were
18 surgical complications included partial or total flap necrosis, haematoma, flap infection, ecchymosis and dehiscence. Medical complications occurred in 20 of 161 procedures (12.4\%). 7 patients had both surgical complications and medical complications. Specific surgical and medical complications are listed in Table 2.

Table 3: Univariate regression analysis of categorical variables.

\begin{tabular}{|c|c|c|c|c|c|c|c|c|}
\hline \multirow[b]{2}{*}{ Variables } & \multicolumn{2}{|c|}{$\begin{array}{l}\text { Complication } \\
\text { ( } n=31)\end{array}$} & \multicolumn{2}{|c|}{$\begin{array}{l}\text { No Complication } \\
(n=130)\end{array}$} & \multirow[b]{2}{*}{ OR } & \multicolumn{2}{|l|}{$95 \% \mathrm{Cl}$} & \multirow[b]{2}{*}{$P$} \\
\hline & n & $\%$ & n & $\%$ & & Lower & Upper & \\
\hline \multicolumn{9}{|l|}{ Sex: } \\
\hline Female & 14 & 21.5 & 51 & 78.5 & & & & 0.546 \\
\hline Male & 17 & 17.7 & 79 & 82.3 & 0.784 & 0.356 & 1.727 & \\
\hline \multicolumn{9}{|l|}{ ASA score: } \\
\hline II & 7 & 8.0 & 81 & 92.0 & & & & $<0.001^{*}$ \\
\hline III & 24 & 32.9 & 49 & 67.1 & 5.668 & 2.273 & 14.131 & \\
\hline \multicolumn{9}{|l|}{ Smoking: } \\
\hline No & 16 & 16.5 & 81 & 83.5 & & & & 0.276 \\
\hline Yes & 15 & 23.4 & 49 & 76.6 & 1.550 & 0.704 & 3.410 & \\
\hline \multicolumn{9}{|l|}{ Comorbidities } \\
\hline No comorbidities & 20 & 18.3 & 89 & 81.7 & & & & $0.017^{*}$ \\
\hline Hypertension & 1 & 4.8 & 20 & 95.2 & 0.223 & 0.028 & 1.756 & 0.154 \\
\hline Diabetes & 1 & 9.1 & 10 & 90.9 & 0.445 & 0.054 & 3.678 & 0.452 \\
\hline Both & 9 & 45.0 & 11 & 55.0 & 3.641 & 1.332 & 9.953 & $0.012^{*}$ \\
\hline \multicolumn{9}{|l|}{ Blood transfusion: } \\
\hline No & 26 & 19.5 & 107 & 80.5 & & & & 0.837 \\
\hline Yes & 5 & 17.9 & 23 & 82.1 & 0.895 & 0.311 & 2.577 & \\
\hline
\end{tabular}

Abbreviations: ASA: American Society of Anesthesiologists; OR: Odds Ratio; Cl: Confidence Interval; ${ }^{*} P<0.05$.

Table 4: Univariate regression analysis of continuous variables.

\begin{tabular}{|c|c|c|c|c|c|c|c|c|}
\hline \multirow[b]{2}{*}{ Variables } & \multicolumn{2}{|c|}{$\begin{array}{l}\text { Complication } \\
\text { ( } \mathrm{n}=\mathbf{3 1})\end{array}$} & \multicolumn{2}{|c|}{$\begin{array}{l}\text { No Complication } \\
(\mathrm{n}=130)\end{array}$} & \multirow[b]{2}{*}{ OR } & \multicolumn{2}{|l|}{$95 \% \mathrm{Cl}$} & \multirow[b]{2}{*}{$P$} \\
\hline & Mean & SD & Mean & SD & & Lower & Upper & \\
\hline Weight, kg & 59.6 & 12.0 & 60.5 & 12.6 & 0.994 & 0.963 & 1.027 & 0.721 \\
\hline Age, yr & 69.4 & 4.0 & 69.0 & 3.5 & 1.029 & 0.925 & 1.145 & 0.599 \\
\hline Preoperative $\mathrm{Hb}, \mathrm{g} / \mathrm{L}$ & 132.1 & 18.3 & 136.4 & 15.6 & 0.983 & 0.960 & 1.008 & 0.178 \\
\hline Preoperative albumin, $\mathrm{g} / \mathrm{L}$ & 40.1 & 3.8 & 40.7 & 3.6 & 0.962 & 0.865 & 1.071 & 0.482 \\
\hline Postoperative Hb, g/L & 97.0 & 10.7 & 112.5 & 15.0 & 0.919 & 0.887 & 0.953 & $<0.001^{*}$ \\
\hline Postoperative albumin, $\mathrm{g} / \mathrm{L}$ & 26.9 & 2.3 & 33.5 & 2.3 & 0.337 & 0.220 & 0.518 & $<0.001^{*}$ \\
\hline Blood loss, ml & 335.5 & 114.2 & 360.0 & 180.3 & 0.999 & 0.996 & 1.002 & 0.469 \\
\hline Crystal during operation, $\mathrm{ml}$ & 2862.9 & 858.4 & 2405.8 & 516.5 & 1.001 & 1.001 & 1.002 & $<0.001^{*}$ \\
\hline Colloid \& FFP during operation, $\mathrm{ml}$ & 637.1 & 354.5 & 849.2 & 415.3 & 0.999 & 0.998 & 1.000 & $0.011^{*}$ \\
\hline Fluids during operation, $\mathrm{ml}$ & 3493.6 & 807.2 & 3207.3 & 655.4 & 1.001 & 1.000 & 1.001 & $0.041^{*}$ \\
\hline Crystal in 24 hours, $\mathrm{ml}$ & 4266.1 & 1016.2 & 4326.9 & 687.7 & 1.000 & 0.999 & 1.000 & 0.688 \\
\hline Colloid in 24 hours, $\mathrm{ml}$ & 637.1 & 354.5 & 776.9 & 403.9 & 0.999 & 0.998 & 1.000 & 0.080 \\
\hline Fluids in 24 hours, $\mathrm{ml}$ & 4890.3 & 1008.2 & 5082.3 & 733.9 & 1.000 & 0.999 & 1.000 & 0.227 \\
\hline
\end{tabular}

Abbreviations: OR: Odds Ratio; Cl: Confidence Interval; SD: Standard Deviation; Hb: Hemoglobin; FFP: Fresh Frozen Plasma; ${ }^{*} P<0.05$. 
Citation: Wang C, Han Z, Cheng S, et al. (2019) Anesthesia-Related Risk Factors for Anterolateral Thigh Free Flap Complications in 161 Elderly Chinese Patients Who Received Oral and Maxillofacial Reconstruction. J Head Neck Surg 2(1):33-37

Table 5: Multivariate logistic regression analysis.

\begin{tabular}{|l|l|l|l|l|}
\hline & \multicolumn{3}{|c|}{ 95\% Cl } & \\
\hline Variables & OR & Lower & Upper & P \\
\hline Postoperative albumin & 0.219 & 0.086 & 0.560 & $0.001^{*}$ \\
\hline ASA & 86.383 & 3.442 & 2167.893 & $0.007^{*}$ \\
\hline Postoperative Hb & 0.829 & 0.686 & 1.002 & 0.052 \\
\hline
\end{tabular}

Abbreviations: OR: Odds Ratio; Cl: Confidence Interval; Hb: Hemoglobin; ${ }^{*} P<0.05$.

\section{Univariate analysis for risk factors of complica- tions}

The results of univariate analysis suggested that, of the factors included in this study, preoperative accompanied diseases including hypertension and diabetes, ASA grade, postoperative hemoglobin level, postoperative albumin level, transfusion volume of crystal, colloid and total liquid during operation were statistically associated with complications ( $P<0.05$ for all factors). Summaries of the univariate analysis were shown in Table 3 and Table 4 . The mean postoperative $\mathrm{Hgb}$ level was $97.0 \mathrm{~g} / \mathrm{L}$ for patients in whom a complication developed compared with $112.5 \mathrm{~g} / \mathrm{L}$ for those without complications. The mean postoperative albumin level was $26.9 \mathrm{~g} / \mathrm{L}$ for those in whom a complication developed compared with $33.5 \mathrm{~g} / \mathrm{L}$ for those without complications. Of the demographic factors analyzed, age and smoking status were not significant predictors of complications.

\section{Multivariate analysis for risk factors of compli- cations}

The results of multivariate analysis showed that the incidence of perioperative complications was significantly associated with ASA grade and postoperative albumin level adjusting for the effect of the other covariates (Table 5).

\section{Discussion}

With the development of the aging society, the proportion of elderly patients with oral and maxillofacial tumors has evidently increased in China [4]. Resection is the most common treatment for those patients. However, it may lead to disfigurement, malformation, and functional lesion. Flap reconstruction of the defects can promote complete or partial recovery of their function and aesthetics, so that these elderly patients can live with greater dignity. The anterolateral thigh free flap was widely used for oral and maxillofacial reconstruction because of its obvious vascular perforation. However, anterolateral thigh free flap reconstruction of oral and maxillofacial defects in elderly patients is complicated and consists of multiple components that may potentially lead to the occurrence of postoperative local or systemic complications. Therefore, the main objective of this retrospective study was to investigate the risk factors, particularly perioperative anesthesia related factors, which contributed to postoperative complications in elderly Chinese patients who underwent reconstruction with ATFF. We conclude that postoperative hypoproteinemia and ASA grade are indepen- dent risk factors for complications of ATFF reconstruction.

\section{Hypoproteinemia}

Albumin plays an important role in the human body, which acts as antithrombotic properties, maintains colloid osmotic pressure, coordinates vascular endothelial integrity membrane integrity and acid-base balance $[5,6]$. Therefore, apart from being employed as an indicator of nutritional status, albumin has also been studied as a prognostic variable for postoperative complications in previous researches. These authors have demonstrated that acute changes in albumin concentrations might identify an invalid reparative reaction, leading to occlusion of the vessel and failure of the free flap [7]. In the present study, we have shown that a postoperative albumin concentration lower than $26.9 \mathrm{~g} / \mathrm{L}$ was an independent risk factor associated with increased flap complications and less satisfied clinical outcomes. As hypoproteinemia may lead to poor resilience, prolonged healing time, and an increased risk of flap reconstruction in elderly patients, maintenance of a normal postoperative serum albumin concentration and postoperative aggressive nutritional supplementation may be benefit for decreasing flap complications.

\section{ASA}

With the increase of aging population, diabetes, hypertension, and heart diseases have become more and more common in elderly patients. In China, because of economic reasons, the medical examination in the elderly population was lacking, particularly those living in the countryside. For this reason, several systemic diseases were lack of proper diagnosis or regular treatment resulting in high ASA grade. With increasing age in elderly patients, the level of tolerance to anesthesia and surgery decreases and the patient's capacity to recover decreases, resulting in prolonged hospitalization and healing and a poor prognosis. In the present study, we identified that ASA grade was an independent risk factor for complications of ATFF reconstruction. This was similar to Fang, et al's., research which also found ASA grade being an independent risk factor for complications of pedicle flaps [8]. Therefore, we suggest taking good control of comorbidities and improving anesthesia tolerance to reduce the risk of complications.

\section{Limitations}

First of all, our study was limited by its retrospective nature, which introduces potential observer and recorder bias into the data collection process. Secondly, the reconstructive surgery in our study was performed by multiple groups of surgeons. Therefore, we may ignore some confounding factors, such as the operating time differs from each groups of surgeons which may results in differences of postoperative flap complications. Finally, the anesthesia team was not consistent throughout the surgical procedure because of the long operating time thus it would lead to small differences in perioperative management possibly. In spite of the existence of these drawbacks, we 
believe that our study provides meaningful clinical data that can sustain in the evidence-based perioperative anesthesia and surgery management of ATFF reconstruction for oral and maxillofacial defects.

\section{Conclusion}

Despite ATFF reconstruction was safe for elderly Chinese patients with oral and maxillofacial tumors, determining perioperative risk factors, especially those anesthesia related that were associated with postoperative flap complications, had important clinical implications. We concluded that postoperative hypoproteinemia and ASA grade were independent risk factors for complications of ATFF reconstruction. On the basis of these findings, efforts should be made to supervise and optimize postoperative albumin and make full preparation before anesthesia to improve surgical and anesthesia tolerance.

\section{References}

1. Coskunfirat OK, Chen HC, Spanio S, et al. (2005) The safety of microvascular free tissue transfer in the elderly population. Plast Reconstr Surg 115: 771-775.

2. Yang R, Lubek JE, Dyalram D, et al. (2014) Head and neck cancer surgery in an elderly patient population: A retrospective review. Int J Oral Maxillofac Surg 43: 1413-1417.

3. Wang C, Fu G, Liu F, et al. (2018) Perioperative risk factors that predict complications of radial forearm free flaps in oral and maxillofacial reconstruction. Br J Oral Maxillofac Surg 56: 514519.

4. Zhang X, Liu F, Lan X, et al. (2014) Combined submandibular gland flap and sternocleidomastoid musculocutaneous flap for postoperative reconstruction in older aged patients with oral cavity and oropharyngeal cancers. World J Surg Oncol 12: 259.

5. Nicholson JP, Wolmarans MR, Park GR (2000) The role of albumin in critical illness. Br J Anaesth 85: 599-610.

6. Neel DR, McClave S, Martindale R (2011) Hypoalbuminaemia in the perioperative period: Clinical significance and management options. Best Pract Res Clin Anaesthesiol 25: 395-400.

7. Shum J, Markiewicz MR, Park E, et al. (2014) Low prealbumin level is a risk factor for microvascular free flap failure. J Oral Maxillofac Surg 72: 169-177.

8. Fang Z, Tian Z, Zhang C, et al. (2016) Risk factors for pedicle flap complications in 251 elderly chinese patients who underwent oral and maxillofacial reconstruction. J Oral Maxillofac Surg 74: 2073-2080. 